\title{
Docosahexaenoic Acid-Induced Lipid Peroxidation and Urinary Excretion of Mercapturic Acid in Rats
}

\author{
Seiji Sekine ${ }^{1,2}$, Kazuhiro Kubo ${ }^{1,3}$, Tadahiro Tadokoro ${ }^{2}$, and Morio Saito ${ }^{1, *}$ \\ ${ }^{1}$ Division of Food Science, Incorporated Administrative Agency, National Institute of Health and Nutrition, 1-23-1 \\ Toyama, Shinjuku-ku, Tokyo 162-8636, Japan \\ ${ }^{2}$ Laboratory of Nourishment Biochemistry, Department of Applied Biology and Chemistry, Tokyo University of Agri- \\ culture, 1-1-1 Sakuragaoka, Setagaya-ku, Tokyo 156-8502, Japan \\ ${ }^{3}$ Nursing Course, Narabunka Women's College, Incorporated Educational Institution, Nara Gakuen, 127 Higashi- \\ naka, Yamatotakada-shi, Nara 635-8530, Japan
}

Received 20 December, 2005; Accepted 20 February, 2006

\begin{abstract}
Summary We hypothesized a suppressive mechanism for dietary docosahexaenoic acid (DHA)-induced tissue lipid peroxidation in which the degradation products, especially aldehydic compounds, are conjugated with glutathione (GSH) through catalysis by glutathione S-transferases (GSTs), and then excreted into urine as mercapturic acids. Sprague-Dawley rats were fed a diet containing DHA ( $8.4 \%$ of total energy) for 31 days. Lipid peroxides in the liver and kidney, liver GST and urinary excretion of mercapturic acid were measured. The lipid peroxide levels in the liver and kidney except the liver aldehydic compounds were higher, and the urinary excretion of mercapturic acid also tended to be higher in the DHA-fed rats although the activity of GST was not increased after DHA intake. We presume from our results that a proportion of the lipid peroxidation-derived aldehydic degradation products might be excreted into urine as mercapturic acid after intake of DHA, thus suppressing the accumulation of aldehydic products in tissues, particularly in the liver.
\end{abstract}

\section{Key Words: Docosahexaenoic Acid, Lipid peroxide, Aldehyde, Glutathione S-transferase,} Mercapturic acid

\section{Introduction}

Docosahexaenoic acid (22:6n-3; DHA) together with eicosapentaenoic acid (20:5n-3; EPA) is the predominant n-3 polyunsaturated fatty acid (PUFA) in fish oils. Consumption of fish oils is particularly associated with a low incidence of atherosclerosis and cardiovascular disease, and this prophylactic effect is attributed to n-3 PUFAs, such as EPA

\footnotetext{
*To whom correspondence should be addressed.

Tel: +81-3-3203-5601 Fax: +81-3-3203-7584

E-mail: msaito@nih.go.jp

Present affiliation: Seiji Sekine, Division of Healthcare Science, Research Laboratory, The Nisshin Oillio Group, Ltd., 1 Shinmeicho, Yokosuka-shi, Kanagawa, 239-0832, Japan.
}

and DHA [1-5]. de Urquiza et al. [6] have recently reported that DHA may influence neural function through activation of a retinoid $\mathrm{X}$ receptor signaling pathway in brain tissue.

However, DHA is very prone to lipid peroxidation because of its unstable chemical structure with six double

\footnotetext{
Abbreviations: ALDH, aldehyde dehydrogenase; AsA, ascorbic acid; DHA, docosahexaenoic acid; EPA, eicosapentaenoic acid; GSH, glutathione; GST, glutathione S-transferase; 4-HAE, 4hydroxy-2-alkenals; 4-HHE, 4-hydroxy-2-hexenal; 4-HNE, 4hydroxy-2-nonenal; LA, linoleic acid; MDA, malondialdehyde; $\mathrm{PCOOH}$, phosphatidylcholine hydroperoxide; $\mathrm{PEOOH}$, phosphatidylethanolamine hydroperoxide; PUFA, polyunsaturated fatty acid; TBA-reactive substances, thiobarbituric acid-reactive substances; Vit. E, vitamin E.
} 
bonds. In previous studies, we showed that DHA ingestion [7-11], similar to fish oil ingestion [12-15], enhanced the susceptibility of rat liver and kidney to lipid peroxidation and increased the requirement for vitamin E (Vit. E). The enhancement was a function of dietary DHA levels and was thought to be attributable to the substitution of membrane fatty acids with highly unsaturated DHA. However, we found that DHA ingestion did not increase the end products of lipid peroxidation, such as lipofuscin, in the liver of rats fed a physiological requirement level of Vit. E $[8,10,11]$. We hypothesized that there are primarily two suppressive mechanisms. One is an antioxidative mechanism that suppresses the generation of lipid peroxides, and the other is a detoxification and/or excretion mechanism that suppresses the accumulation of lipid peroxides. The former mechanism is thought to be exerted through increases in ascorbic acid (AsA) and glutathione (GSH) induced by DHA intake [8, 9, 10], which potentiates reductive recycling of Vit. E, thus maintaining antioxidative potency. However, the DHAinduced generation of tissue lipid peroxides was not suppressed further even after higher doses of Vit. E [7, 8], or after higher doses of AsA and methionine $[10,11]$. Methionine is necessary for the synthesis of GSH via cysteine. Accordingly, the antioxidative potency exerted through Vit. E, AsA and GSH is not enough to suppress the generation of lipid peroxides. Additionally, ingestion of DHA did not increase liver glutathione reductase and glutathione peroxidase activities, even though the tissue lipid peroxide levels in DHA-fed rats were higher [8, 9]. These observations suggest that some mechanisms other than antioxidants and antioxidant enzymes are induced in order to suppress the accumulation of lipid peroxides after DHA ingestion.

When 4-hydroxy-2-hexenal (4-HHE) [17] and 4-hydroxy2-nonenal (4-HNE) [18] as secondary degradation products of lipid peroxidation are injected into the blood stream of rats, the mercapturic acids (acetylcysteine conjugates) derived metabolically from conjugates of the aldehydes with GSH are detected in the urine. Therefore, we presumed that detoxification and/or excretion of reactive aldehydes derived from DHA-induced lipid peroxidation is a second mechanism for suppressing the accumulation of lipid peroxides, in which glutathione S-transferases (GSTs) firstly catalyze the conjugation of lipid peroxidation-derived aldehydes with GSH. In addition, we hypothesized that another mechanism involving dietary DHA-induced biotransformation mediated by aldehyde dehydrogenases (ALDHs) detoxifies reactive aldehydes, resulting in formation of their carboxylic acids [18].

Clarification of the mechanisms that suppress dietary DHA-induced lipid peroxidation is important not only for reasons of safety, but also to formulate a means of efficiently enhancing the physiological efficacy of n-3 PUFAs. In this study, therefore, we focused in particular on DHA intakeinduced detoxification and/or excretion mechanisms for suppressing the accumulation of lipid peroxides, particularly reactive aldehydes, thus suppressing formation of their end products, in rats.

\section{Materials and Methods}

\section{Animals and Diets}

The experimental procedures used in this study met the guidelines of the animal handling committee in Incorporated Administrative Agency, National Institute of Health and Nutrition (Tokyo, Japan).

Male Sprague-Dawley rats (Japan SLC, Hamamatsu, Japan), 5 weeks of age and weighing 100-125 g, were housed individually in stainless-steel wire-bottomed cages kept at a constant temperature of $22 \pm 1^{\circ} \mathrm{C}$ and humidity of $50-60 \%$ with a $12 \mathrm{~h}$ light-dark cycle. The composition of the experimental diets, based on the AIN-76 purified diet for rats [19-21], is shown in Table 1. Six rats each were assigned to the control and DHA groups. The diet and water were consumed ad libitum for 31 days. The dietary lipid level was $100 \mathrm{~g} / \mathrm{kg}$ diet. DHA ethyl esters (93.0\% pure) prepared from orbital fat of tuna was donated by Maruha Corporation (Tsukuba, Japan). In the control diet, the level of linoleic acid (18:2n-6; LA) was $11.8 \%$ of total energy. In the DHA-containing diet, the levels of LA and DHA were 3.0 and $8.4 \%$ of total energy, respectively. The proportion of total PUFA was at almost the same level in the control and DHA-containing diets. The Vit. E content as $R R R-\alpha-$ tocopherol equivalent of the experimental diets was $70 \mathrm{mg} / \mathrm{kg}$ diet, which is a physiological requirement level of Vit. E. The biopotency ratio of $R R R-\alpha-, R R R-\beta-, R R R-\gamma-$ and $R R R-\delta-$ tocopherols was 100:25:5:0.1, respectively, in the calculation of $R R R$ - $\alpha$-tocopherol equivalent [22]. All-rac- $\alpha$-tocopheryl acetate ( $>99 \%$ pure) was used to adjust the Vit. E content in the diets. To prevent the autoxidation of DHA in the diet, the diet was prepared beforehand without adding DHA and stored at $-20^{\circ} \mathrm{C}$. DHA was stored at $-80^{\circ} \mathrm{C}$. They were mixed every day immediately before feeding.

During the experimental period, each diet was made available to the rats in the evening and was removed the next morning. After being deprived of food overnight, the rats were sacrificed by decapitation. The liver and kidney were promptly excised, washed with isotonic saline and weighed. The liver was then perfused with ice-cold isotonic saline via the portal vein. The liver and kidney samples were stored at $-80^{\circ} \mathrm{C}$ until used for the analysis.

Analytical procedures
Phosphatidylcholine hydroperoxide (PCOOH) and phos-
phatidylethanolamine hydroperoxide (PEOOH)_Liver
samples were assayed according to the method of Miyazawa 
Table 1. Composition of experimental diets ( $\mathrm{g} / \mathrm{kg} \mathrm{diet}$ ) and fatty acid composition $(\mathrm{g} / 100 \mathrm{~g})$ of dietary lipids given to rats. $^{\text {a }}$

\begin{tabular}{|c|c|c|}
\hline Group & Control & DHA \\
\hline & \multicolumn{2}{|c|}{$\%$ total energy } \\
\hline Linoleic acid & 11.8 & 3.0 \\
\hline \multirow[t]{2}{*}{ DHA } & 0 & 8.4 \\
\hline & \multicolumn{2}{|c|}{$\mathrm{g} / \mathrm{kg}$ diet } \\
\hline Basic components ${ }^{\mathrm{b}}$ & 900 & 900 \\
\hline Test lipids $^{\mathrm{c}}$ & 100 & 100 \\
\hline Stripped corn oil & 100 & 36 \\
\hline Olive oil & 0 & 20 \\
\hline DHA concentrate ${ }^{d}$ & 0 & 44 \\
\hline Fatty acids & \multicolumn{2}{|c|}{$\mathrm{g} / 100 \mathrm{~g}$} \\
\hline $16: 0$ & 10.3 & 5.5 \\
\hline $18: 0$ & 1.8 & 1.6 \\
\hline $18: 1(n-9)$ & 30.5 & 35.3 \\
\hline 18:2 (n-6) LA & 54.5 & 13.8 \\
\hline $18: 3(n-3)$ & 0.8 & 0.4 \\
\hline $20: 4(n-6)$ & ND & 0.7 \\
\hline 20:5 (n-3) EPA & ND & 0.5 \\
\hline $22: 5(n-6)$ & ND & 0.2 \\
\hline $22: 5(n-3)$ & ND & 0.7 \\
\hline 22:6 (n-3) DHA & ND & 39.0 \\
\hline Others & 2.1 & 2.3 \\
\hline PUFA & 55.4 & 55.3 \\
\hline
\end{tabular}

a The energy density of all diets was $17.4 \mathrm{MJ} / \mathrm{kg} \operatorname{diet}(4160 \mathrm{kcal} /$ $\mathrm{kg}$ ), using Atwater energy factors for energy calculation [19]. The vitamin $\mathrm{E}$ content as $R R R$ - $\alpha$-tocopherol equivalent of the experimental diets was $70 \mathrm{mg} / \mathrm{kg}$ diet. ND, not detectable.

$\mathrm{b}$ The basic components of the diet given to all the groups were as follows: casein, $200.0 \mathrm{~g}$; DL-methionine, $3.0 \mathrm{~g}$; cornstarch, $150.0 \mathrm{~g}$; sucrose, $225.0 \mathrm{~g}$; glucose, $225.0 \mathrm{~g}$; cellulose powder, 50.0 g; AIN-76 vitamin mixture [20,21], 10.0 g; AIN-76 mineral mixture [20], 35.0; choline bitartrate, $2 \mathrm{~g}$.

${ }^{c}$ Fat energy percentage is $21.6 \%$ of total energy.

$\mathrm{d}$ The purity of the DHA ethyl esters was $93.0 \%$.

et al. [23], that is, phospholipids were extracted from the homogenate by the method of Bligh and Dyer [24] and the $\mathrm{PCOOH}$ and $\mathrm{PEOOH}$ were analyzed with chemiluminescence-HPLC system.

Thiobarbituric acid-reactive substances (TBA-reactive substances)-The tissue Thiobarbituric acid-reactive substances (TBA-reactive substances) were measured according to the method of Ohkawa et al. [25] with a minor modification, in which butylated hydroxytoluene was added to the reaction mixture at a final concentration of $0.45 \mathrm{mM}$. TBA-reactive substances are expressed in terms of the malondialdehyde (MDA) equivalent.

Malondialdehyde (MDA) + 4-hydroxy-2-alkenals (4-HAE) -
The tissue free MDA + 4-HAE were determined using commercial BIOXYTECH ${ }^{\circledR}$ LPO-586 ${ }^{\mathrm{TM}}$ assay kit (OXIS International Inc., Portland, OR, USA). The LPO-586 method is designed to assay MDA in conjunction with 4HAE in methanesulfonic acid. The steady-state level of 4HNE which is one of the 4-HAE is shown to increase under pathophysiological states associated with oxidative stress [26].

Vitamin $E$ ( $\alpha$-Tocopherol) $-\alpha$-Tocopherol concentration in the test lipids was analyzed by HPLC [27].

GST and ALDH activities - GST activity was determined by the method of Jensson et al. [28]. ALDH activity was determined by the method of Pietruszko and Yonetani [29]. The protein content was measured by the method of Lowry et al. [30].

Urinary mercapturic acid excretion-The urinary mercapturic acid level was analyzed by HPLC after the extraction by the method of Kress and Pentz [31].

Fatty acid composition - Fatty acid methyl esters of dietary lipids were prepared and analyzed by GLC according to a previous study [10].

Statistical analysis-Significant difference between the mean values of the control and DHA groups was evaluated by Student's $t$-test. The limit of significance was set at $p<0.05$.

\section{Results}

The rats consumed 16.1-16.8 g food/d and gained 5.3$5.8 \mathrm{~g} / \mathrm{d}$ over the 31 days of the experimental period. There were no significant differences in food intake, body-weight gain and liver and kidney weights between the control and DHA groups. (data not shown).

The levels of PCOOH, PEOOH and MDA + 4-HAE in the liver did not differ significantly between the two groups as shown in Table 2. The TBA-reactive substances level was significantly higher in the liver of the DHA group than that of the control. The activities of liver GST and ALDH were not significantly different between the two groups. The levels of kidney TBA-reactive substances and MDA + 4-HAE were significantly higher in the DHA group than in the control group (Table 2). The urinary excretion of mercapturic acid on day 28 tended to be higher in the DHA group but the difference was not statistically significant due to considerable scatter in the data (Table 2).

\section{Discussion}

In this study, we focused in particular on detoxification and/ or excretion mechanisms in which lipid peroxidationderived degradation products, particularly reactive aldehydes, are conjugated with GSH, metabolized to mercapturic acid and excreted into urine, thus suppressing dietary DHA-induced 
Table 2. Liver and kidney lipid peroxide levels, liver GST and ALDH activities, and urinary excretion of mercapturic acid in rats. ${ }^{\mathrm{a}}$

\begin{tabular}{lrr}
\hline Group & \multicolumn{1}{c}{ Control } & \multicolumn{1}{c}{ DHA } \\
\hline Liver & & \\
PC-OOH (pmol/g) & $15.25 \pm 6.19$ & $17.06 \pm 6.80$ \\
PE-OOH (pmol/g) & $5.51 \pm 2.46$ & $6.25 \pm 3.19$ \\
TBARS (nmol/g) & $72.29 \pm 4.93^{*}$ & $130.26 \pm 7.67$ \\
MDA + 4-HAE (nmol/g) & $3.17 \pm 0.74$ & $3.21 \pm 0.33$ \\
GST (unit/mg protein) & $1.63 \pm 0.09$ & $1.55 \pm 0.11$ \\
ALDH (unit/mg protein) & $26.68 \pm 4.99$ & $29.2 \pm 7.44$ \\
Kidney & & \\
TBARS (nmol/g) & $108.42 \pm 15.84 *$ & $129.39 \pm 16.23$ \\
MDA + 4-HAE (nmol/g) & $2.37 \pm 0.30 *$ & $2.91 \pm 0.29$ \\
Urinary mercapturic acid & & \\
Day 28 & & \\
( $\mu$ mol/day) & $3.11 \pm 1.93$ & $5.25 \pm 3.35$ \\
\hline Abbreviations: ALDH, aldehyde & & \\
\hline
\end{tabular}

Abbreviations: ALDH, aldehyde dehydrogenase; GST, glutathione S-transferase; MDA + 4-HAE, malondialdehyde + 4-hydroxy-2alkenals; $\mathrm{PC}-\mathrm{OOH}$, phosphatidylcholine hydroperoxide; $\mathrm{PE}-\mathrm{OOH}$, phosphatidylethanolamine hydroperoxide; TBARS, thiobarbituric acid-reactive substances.

a Values are mean $\pm \mathrm{SD}, \mathrm{n}=6$.

* Mean values are significantly different from the DHA group (Student's $t$-test), $p<0.05$.

accumulation of tissue aldehydic compounds, and eventually the end products of lipid peroxidation, such as lipofuscin, as observed previously $[8,10,11]$. To prove this hypothetical scheme, we fed rats a very high level of DHA ( $8.4 \%$ of total energy) which is actually hard to ingest in our daily life. But such a model system is in particular necessary to highlight the in vivo response and to detect it.

The levels of PCOOH, PEOOH and free MDA + 4-HAE in the liver did not differ significantly, but the TBA-reactive substances level was significantly higher after DHA intake (Table 2). Various and complex mixtures of compounds derived from in vivo lipid peroxidation, and also from thiobarbituric acid-reactive precursors which degrade under the acidity and heating used in the assay, react with thiobarbituric acid. These compounds include lipid hydroperoxides, hydroperoxyendoperoxides, malondialdehyde, alkenals, alkadienals, and so forth. To reduce the formation of artifacts as least as possible in the TBA-reactive substances assay, we added butylated hydroxytoluene to the reaction mixture. It was shown that in TBA-reactive substances assay in fat from chicken meat, $125 \mu \mathrm{g}$ of butylated hydroxytoluene/mg of fat was enough to prevent autoxidation during the heating step of the assay [32]. Taking the present assay samples prone to autoxidation in the assay into account, we added higher amount of butylated hydroxytoluene (final concentration is about $180 \mu \mathrm{g} / \mathrm{mg}$ of fat) in the determination of TBA- reactive substances levels.

On the other hand, the LPO-586 ${ }^{\mathrm{TM}}$ (OXIS) assay kit specifically measures free MDA + 4-HAE. One of the reasons why the $\mathrm{PCOOH}$ and $\mathrm{PEOOH}$ levels did not increase in the liver, even after the intake of highly unsaturated DHA, seems to be very rapid progress of lipid peroxidation from the initial stage, when these hydroperoxides are generated, to propagation stage producing TBA-reactive substances, because the proportions of highly unsaturated PUFAs such as EPA, 22:5n-3, and DHA become higher in the liver, and conversely those of LA and arachidonic acid, which have a low degree of unsaturation, become lower after DHA intake, as observed previously $[7,10,33]$. However, free MDA + 4-HAE did not increase even in the propagation stage of lipid peroxidation in the liver. Hence, lipid peroxidation was not promoted sufficiently to produce free aldehydes or free aldehydes produced were efficiently metabolized in the liver and/or excreted from the liver.

Large numbers and species of xenobiotics are conjugated with GSH through catalysis by GSTs in the phase II drugmetabolizing pathways following the phase I pathways catalyzed by cytochrome P-450s in the liver. The conjugates are then transported to the kidney, where glutamic acid and glycine are removed, yielding cysteine conjugates that are further acetylated to mercapturic acids for excretion into urine. When n-3 PUFA-derived 4-HHE [17] and n-6 PUFAderived 4-HNE [18] as secondary degradation products of lipid peroxidation were injected into the blood stream of rats, their mercapturic acid conjugates were detected in the urine. In this study as well, although the difference was not statistically significant, the urinary excretion of mercapturic acid tended to be higher in the DHA-fed rats (Table 2). This observation may support the finding that the lipid peroxidation-derived free MDA + 4-HAE did not increase in the liver of DHA-fed rats. That is, the mercapturic acid conjugates of such aldehydes might be excreted into urine. However, the total activity of GSTs in the liver did not differ significantly between the control and DHA-fed groups (Table 2). Ålin et al. [34] and Hiratsuka et al. [35] reported that GSTA4-4, which present as a very minor GST protein in rat liver, exhibited extremely high catalytic activity towards 4-HNE. When GSTs in rat liver were separated by the chromatography on DEAE-cellulose column, GST A4-4 (also called GST $8-8)$ activity $(60 \mu \mathrm{mol} / \mathrm{min})$ was much lower than other GSTs activity $(4460 \mu \mathrm{mol} / \mathrm{min})$ [34]. Accordingly, even if the total activity of GSTs does not change in rats fed DHA, GSTs could be associated with the excretion of lipid peroxidation-derived reactive aldehydes including 4-HNE and even 4-HHE. The statistically insignificant difference in the level of mercapturic acid excretion between the control group rich in n-6 PUFAs and DHA-fed group rich in n-3 PUFAs might be due to the 
difference in the substrate specificity of GST A4-4 for 4HNE and 4-HHE, as described below.

Hubatsch et al. [36] reported that the specific activity of GST A4-4 with 4-HNE derived from oxidation of $n-6$ PUFAs was higher than that with 4-HHE derived from oxidation of n-3 PUFAs. Fukuda et al. [37] also obtained similar results. Hence, the excretion of mercapturic acid into urine might have been higher in the n-6 LA-containing diet groups. Therefore, the absence of clear difference in the urinary excretion of mercapturic acid seems to reflect the difference in substrate specificity between 4-HNE and 4HHE with GSH for GST and also in the extent of oxidation of PUFAs in cell membranes. Studies to explore this precise mechanism are under way.

The levels of kidney lipid peroxides were significantly higher in the DHA group than in the control group, but the differences of the levels between the two groups were small. Considering the PUFA composition of the kidney [7, 10, 33 , the proportions of n-6 PUFAs with a low degree of unsaturation are generally higher, and that of highly unsaturated DHA is lower, than in the liver. In addition, high retroconversion from DHA to EPA was shown in the kidney $[7,10,33]$. Furthermore, although the Vit. E level in the kidney becomes lower with DHA intake, the extent of the decrease is small compared with that in the liver, as already observed $[7,8,9,16]$. Hence, the requirement for Vit. E is low in the kidney. Because of these characteristic profiles and the plausible difference in antioxidant potential, the difference of the lipid peroxide levels in the kidney might have been small between the two groups.

The phospholipid hydroperoxides and aldehydic products were not increased in the liver even after a very high level of DHA intake. Accordingly, we presume from the results obtained herein that a proportion of the lipid peroxidationderived aldehydic degradation products following intake of DHA might be excreted into urine as mercapturic acid via conjugation by GST, thus suppressing accumulation of aldehydic products in tissues, particularly in the liver. Such detoxification and/or excretion mechanisms might play an important role in suppressing tissue damages to occur.

\section{Acknowledgments}

We thank Maruha Corporation, Japan, for the generous gift of the DHA ethyl ester concentrate.

\section{References}

[1] Bang, H.O. and Dyerberg, J.: Plasma lipids and lipoproteins in Greenlandic west coast Eskimos. Acta. Med. Scand., 192, 85-94, 1972.

[2] Herold, P.M. and Kinsella, J.E.: Fish oil consumption and decreased risk of cardiovascular disease: a comparison of findings from animal and human feeding trials. Am. J. Clin. Nutr., 43, 566-598, 1986.

[3] Harris, W.S.: Fish oils and plasma lipid and lipoprotein metabolism in humans: a critical review. J. Lipid Res., 30, 785-807, 1989.

[4] Simopoulos, A.P.: Omega-3 fatty acids in health and disease and in growth and development. Am. J. Clin. Nutr., 54, 438463, 1991.

[5] Simopoulos, A.P.: Essential fatty acids in health and chronic disease. Am. J. Clin. Nutr., 70, 560S-569S, 1999.

[6] de Urquiza, A.M., Liu, S., Sjöberg, M., Zetterström, R.H., Griffiths, W., Sjövall, J., and Perlmann, T.: Docosahexaenoic acid, a ligand for the retinoid $\mathrm{X}$ receptor in mouse brain. Science, 290, 2140-2144, 2000.

[7] Kubo, K., Saito, M., Tadokoro, T., and Maekawa, A.: Dietary docosahexaenoic acid dose not promote lipid peroxidation in rat tissue to the extent expected from peroxidizability index of tissue total lipid. Biosci. Biotech. Biochem., 62, 16981706, 1998.

[8] Kubo, K., Saito, M., Tadokoro, T., and Maekawa, A.: Preferential incorporation of docosahexaenoic acid into nonphosphorus lipids and phosphatidylethanolamine protects rats from dietary DHA-stimulated lipid peroxidation. J. Nutr., 130, 1749-1759, 2000.

[9] Saito, M. and Kubo, K.: Relationship between tissue lipid peroxidation and peroxidizability index after $\alpha$-linolenic, eicosapentaenoic, or docosahexaenoic acid intake in rats. $\mathrm{Br}$. J. Nutr., 89, 19-28, 2003.

[10] Sekine, S., Kubo, K., Tadokoro, T., Maekawa, A., and Saito, M.: Dietary docosahexaenoic acid-induced production of tissue lipid peroxides is not suppressed by higher intake of ascorbic acid in genetically scorbutic Osteogenic Disorder Shionogi/Shi-od/od rats. Br. J. Nutr., 90, 385-394, 2003.

[11] Sekine, S., Kubo, K., Tadokoro, T., and Saito, M.: Dietary docosahexaenoic acid-induced generation of liver lipid peroxides is not suppressed further by elevated levels of glutathione in ODS rats. Nutrition, 22, 385-394, 2006.

[12] Hammer, C.T. and Wills, E.D.: The role of lipid components of the diet in the regulation of the fatty acid composition of the rat liver endoplasmic reticulum and lipid peroxidation. Biochem. J., 174, 585-593, 1978.

[13] Kobatake, Y., Hirahara, F., Innami, S., and Nishide, E.: Dietary effect of n-3 type polyunsaturated fatty acids on serum and liver lipid levels in rats. J. Nutr. Sci. Vitaminol., 29, 11-21, 1983.

[14] Mouri, K., Ikesue, H., Esaka, T., and Igarashi, O.: The influence of marine oil intake upon levels of lipids, $\alpha$ tocopherol and lipid peroxidation in serum and liver of rats. J. Nutr. Sci. Vitaminol., 30, 307-318, 1984.

[15] Song, J.H., Fujimoto, K., and Miyazawa, T.: Polyunsaturated (n-3) fatty acids susceptible to peroxidation are increased in plasma and tissue lipids of rats fed docosahexaenoic acidcontaining oils. J. Nutr., 130, 3028-3033, 2000.

[16] Kubo. K., Saito. M., Tadokoro. T., and Maekawa. A.: Changes in susceptibility of tissues to lipid peroxidation after ingestion of various levels of docosahexaenoic acid and vitamin E. Br. J. Nutr., 78, 655-669, 1997. 
[17] Winter, C.K., Segall, H.J., and Jones, A.D.: Distribution of trans-4-hydroxy-2-hexenal and tandem mass spectrometric detection of its urinary mercapturic acid in rat. Drug Metab. Dispos., 15, 608-612, 1987.

[18] Alary, J., Bravais, F., Cravedi, J-P., Debrauwer, L., Rao, D., and Bories, G.: Mercapturic acid conjugates as urinary end metabolites of the lipid peroxidation products 4-hydroxy-2nonenal in the rat. Chem. Res. Toxicol., 8, 34-39, 1995.

[19] Atwater, W.O.: Principles of nutrition and nutritive value of food. US Dep. Agric. Farmers' Bull., 142, 48, 1902.

[20] American Institute of Nutrition: Second report of ad hoc committee on standards for nutritional studies. J. Nutr., 110, 1726, 1980.

[21] American Institute of Nutrition: Report of the American Institute of Nutrition ad hoc committee on standards for nutritional studies. J. Nutr., 107, 1340-1348, 1977.

[22] Mino, M., Tamai, H., Yasuda, C., Igarashi, O., Hayashi, M., Hirahara, F., Katsui, G., and Kijima, S.: Biopotencies of tocopherol analogues as determined by dialuric acid-induced hemolysis in rats. Vitamins (Japan), 62, 241-246, 1988.

[23] Miyazawa, T., Fujimoto, K., Suzuki, T., and Yasuda, K.: Determination of phospholipid hydroperoxides using luminol chemiluminescence-high-performance liquid chromatography. Methods Enzymol., 233, 324-332, 1994.

[24] Bligh, E.G. and Dyer, W.J.: A rapid method of total lipid extraction and purification. Can. J. Biochem. Physiol., 37, 911-917, 1959.

[25] Ohkawa, H., Ohnishi, N., and Yagi, K.: Assay for lipid peroxides in animal tissues by thiobarbituric acid reaction. Anal. Biochem., 95, 351-358, 1979.

[26] Uchida, K.: Protein-bound 4 hydroxy-2-nonenal as a marker of oxidative stress. J. Clin. Biochem. Nutr., 36, 1-10, 2005.

[27] Saito, M., Nakatsugawa, K., Oh-hashi, A., Nishimuta, M., and Kodama, N.: Comparison of vitamin E levels in human plasma, red blood cells, and platelets following varying intakes of vitamin E. J. Clin. Biochem. Nutr., 12, 5968,1992.

[28] Jennson, H., Ålin, P., and Mannervik, B.: [63] Glutathione transferase isozymes from rat liver cytosol. Methods
Enzymol., 113, 504-507, 1985.

[29] Pietruszko, R. and Yonetani, T.: Aldehyde dehydrogenases from liver. Methods Enzymol., 71, 772-781, 1981.

[30] Lowry, O.H., Rosebrough, N.J., Farr, A.L., and Randall, R.J.: Protein measurement with the Folin phenol reagent. J. Biol. Chem., 193, 265-275, 1951.

[31] Kress, G. and Pentz, R.: A sensitive method for the determination of urinary mercapturic acids for use in biological monitoring. Arch. Toxicol. Suppl., 13, 104-107, 1989.

[32] Pikul, J., Leszczynski, D.E., and Kummerow, F.A.: Elimination of sample autoxidation by butylated hydroxytoluene additions before thiobarbituric acid assay for malonaldehyde in fat from chiken meat. J. Agric. Food Chem., 31, 1338-1342, 1983.

[33] Saito, M., Ueno, M., Kubo, K., and Yamaguchi, M.: Doseresponse effect of dietary docosahexaenoic acid on fatty acid profiles of serum and tissue lipids in rats. J. Agric. Food Chem., 46, 184-193, 1998.

[34] Ålin, P., Jensson, H., Cederlund, E., Jörnvall, H., and Mannervik, B.: Cytosolic glutathione transferases from rat liver: primary structure of class alpha glutathione transferase 8-8 and characterization of low-abundant class $\mathrm{Mu}$ glutathione transferases. Biochem. J., 261, 531-539, 1989.

[35] Hiratsuka, A., Tobita, K., Saito, H., Sakamoto, Y., Nakano, H., Ogura, K., Nishiyama, T., and Watanabe, T.: (S)Preferential detoxification of 4-hydroxy-2(E)-nonenal enantiomers by hepatic glutathione S-transferase isoforms in guinea-pigs and rats. Biochem. J., 355, 237-244, 2001.

[36] Hubatsch, I., Ridderström, M., and Mannervik, B.: Human glutathione transferase A4-4: an alpha class enzyme with high catalytic efficiency in the conjugation of 4hydroxynonenal and other genotoxic products of lipid peroxidation. Biochem. J., 330, 175-179, 1998.

[37] Fukuda, A., Nakamura, Y., Ohigashi, H., Osawa, T., and Uchida, K.: Cellular response to the redox active lipid peroxidation products: induction of glutathione S-transferase P by 4-hydroxy-2-nonenal. Biochem. Biophys. Res. Commun., 236, 505-509, 1997. 